\title{
ON SOME PROPERTIES OF PBZ*-LATTICES
}

\author{
ROBERTO GIUNTINI, ANTONIO LEDDA, FRANCESCO PAOLI
}

\begin{abstract}
We continue the algebraic investigation of $P B Z^{*}$-lattices, a notion introduced in [12] in order to obtain insights into the structure of certain algebras of effects of a Hilbert space, lattice-ordered under the spectral ordering.

Keywords: Orthomodular lattice; PBZ*-lattice; Brouwer-Zadeh lattice; Kleene lattice; Unsharp quantum theory; Effect; Spectral ordering.
\end{abstract}

\section{INTRODUCTION}

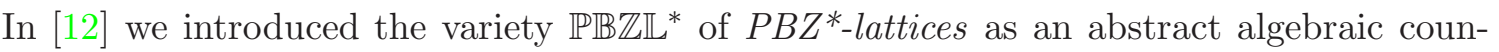
terpart of the structure

$$
\left\langle\mathcal{E}(\mathbf{H}), \wedge, \vee,^{\prime}, \sim, \mathbb{O}, \mathbb{I}\right\rangle,
$$

where:

- $\mathcal{E}(\mathbf{H})$ is the set of all effects of a given complex separable Hilbert space;

- $\wedge$ and $\vee$ are the meet and the join, respectively, of the spectral ordering $\leq_{s}$ so defined $^{1}$ for all $E, F \in \mathcal{E}(\mathbf{H})$ :

$$
E \leq_{s} F \text { iff } \forall \lambda \in \mathbb{R}: M^{F}(\lambda) \leq M^{E}(\lambda),
$$

where for any effect $E, M^{E}$ is the unique spectral family [17, Ch. 7] such that $E=\int_{-\infty}^{\infty} \lambda d M^{E}(\lambda)$ (the integral is here meant in the sense of norm-converging Riemann-Stieltjes sums [19, Ch. 1]);

- $\mathbb{O}$ and $\mathbb{I}$ are the null and identity operators, respectively;

- $E^{\prime}=\mathbb{I}-E$ and $E^{\sim}=P_{\operatorname{ker}(E)}$ (the projection onto the kernel of $E$ ).

This class of algebras is further motivated, from a physical viewpoint, by the fact that it reproduces at an abstract level the "collapse" of several notions of sharp physical property that is observed in the concrete physical model over $\mathcal{E}(\mathbf{H})$; from an algebraic viewpoint, moreover, it can be viewed as an unsharp generalisation of orthomodular lattices that also

\footnotetext{
${ }^{1}$ That the spectral ordering is indeed a lattice ordering has been essentially shown by Olson [18] and de Groote [14], who also proved that it coincides with the more familiar ordering of effects induced via the trace functional when both orderings are restricted to the set of projection operators of the same Hilbert space. The same ordering has also been given an algebraic treatment, in a different context, in [10].
} 
covers certain expansions of Kleene lattices — and, consequently, may have some potential interest for many-valued logics of partial information [1].

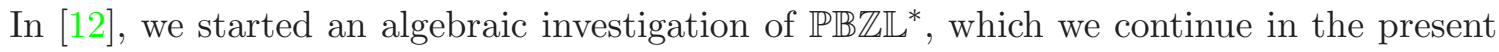
paper. In Section 2, the main definitions and results of [12] are summarised, with an eye to making this work reasonably self-contained. In Section 3, we improve on the description of the lattice of subvarieties of $\mathbb{P B Z \mathbb { L }}{ }^{*}$ given in the same paper. The main result of this section is a representation theorem for subdirectly irreducible antiortholattices (see below for a definition) in terms of twist structures over bounded lattices, which is applied later on to identify a suitable set of generators for the distributive subvariety of the variety $V(\mathbb{A O L})$ generated by antiortholattices. We also show that the distributive subvariety of $V(\mathbb{A O L})$ satisfying the De Morgan law for the intuitionistic complement $\sim$ is generated by the 5-element antiortholattice chain, and we simplify an already known equational basis for the subvariety of $V(\mathbb{A O L})$ generated by the 3-element antiortholattice chain. In Section 4 we investigate horizontal sums of $\mathrm{PBZ}^{*}$-lattices, showing that the variety generated by subdirectly irreducible PBZ*-lattices which are a horizontal sum of Boolean algebras and an ortholattice chain is generated by its finite members ${ }^{2}$.

\section{Preliminaries}

We recap in this section some definition and results (the latter mostly from [12], except when explicitly noted) which will be needed in the sequel.

Definition 2.1. A bounded involution lattice $\mathbf{L}=\left\langle L, \wedge, \vee,^{\prime}, 0,1\right\rangle$ is a pseudo-Kleene algebra in case it satisfies any of the following two equivalent conditions:

(1) for all $a, b \in L$, if $a \leq a^{\prime}$ and $b \leq b^{\prime}$, then $a \leq b^{\prime}$;

(2) for all $a, b \in L, a \wedge a^{\prime} \leq b \vee b^{\prime}$.

The variety of pseudo-Kleene algebras, for which see e.g. [6], is denoted by $\mathbb{P} \mathbb{A}$. Distributive pseudo-Kleene algebras are variously called Kleene lattices or Kleene algebras in the literature. Observe that in [12], embracing the terminological usage from [9, p. 12], pseudo-Kleene algebras were referred to as "Kleene lattices". Here, we switch to the less ambiguous "pseudo-Kleene algebras", following the suggestion of a referee.

In unsharp quantum logic, there are several competing purely algebraic characterisations of sharp effects [9, Ch. 7]. A quantum effect or property is usually called sharp if it satisfies the noncontradiction principle:

Definition 2.2. Let $\mathbf{L} \in \mathbb{P} \mathbb{K}$.

(1) An element $a \in L$ is said to be Kleene-sharp iff $a \wedge a^{\prime}=0 . S_{K}(L)$ denotes the class of Kleene-sharp elements of $\mathbf{L}$.

\footnotetext{
${ }^{2}$ We acknowledge here G. Cattaneo's insightful comments on [12], which we received after the paper itself had gone into its production stage.
} 
(2) An ortholattice is a bounded involution lattice $\mathbf{L}$ such that $S_{K}(L)=L$. The variety of ortholattices is denoted by $\mathbb{O L}$.

Among ortholattices, orthomodular lattices play a crucial role in the standard (sharp) approach to quantum logic. Recall that an orthomodular lattice is an ortholattice $\mathbf{L}$ such that, for all $a, b \in L$, if $a \leq b$, then $b=\left(b \wedge a^{\prime}\right) \vee a$. The class of orthomodular lattices is actually a variety, hereafter denoted by $\mathbb{O M L}$.

It is well-known that an ortholattice $\mathbf{L}$ is orthomodular if and only if, for all $a, b \in L$, if $a \leq b$ and $a^{\prime} \wedge b=0$, then $a=b$. The right-to-left direction of this equivalence fails in the wider setting of bounded involution lattices. This justifies the following definition, aimed at recapturing at least in part the force of the orthomodular condition in bounded involution lattices that may contain Kleene-unsharp elements.

Definition 2.3. An algebra $\mathbf{L}$ with a bounded involution lattice term reduct is said to be paraorthomodular iff, for all $a, b \in L$ :

$$
\text { if } a \leq b \text { and } a^{\prime} \wedge b=0 \text {, then } a=b .
$$

It turns out that the class of paraorthomodular pseudo-Kleene algebras is a proper quasivariety, whence we cannot help ourselves to the strong universal algebraic properties that characterise varieties. It is then natural to wonder whether there exists an expansion of the language of bounded involution lattices where the paraorthomodular condition can be equationally recovered. The appropriate language expansion is provided by including an additional unary operation and moving to the $\langle 2,2,1,1,0,0\rangle$ type, already familiar to unsharp quantum logicians and algebraists from the investigation of Brouwer-Zadeh lattices (see [5] or [9, Ch. 4.2]).

Definition 2.4. A Brouwer Zadeh lattice (or BZ-lattice) is an algebra

$$
\mathbf{B}=\left\langle B, \wedge, \vee,^{\prime}, \sim, 0,1\right\rangle
$$

of type $\langle 2,2,1,1,0,0\rangle$, such that:

(1) $\left\langle B, \wedge, \vee,^{\prime}, 0,1\right\rangle$ is a pseudo-Kleene algebra;

(2) for all $a, b \in B$, the following conditions are satisfied:

$$
\begin{array}{ll}
\text { (i) } a \wedge a^{\sim}=0 ; & \text { (ii) } a \leq a^{\sim \sim} \\
\text { (iii) } a \leq b \text { implies } \quad b^{\sim} \leq a^{\sim} ; & \text { (iv) } a^{\sim \prime}=a^{\sim \sim} .
\end{array}
$$

The class of all BZ-lattices is a variety, denoted by $\mathbb{B Z L}$; $\mathbb{O L}$ can be identified with the subvariety of $\mathbb{B} \mathbb{Z} \mathbb{L}$ whose relative equational basis w.r.t. $\mathbb{B} \mathbb{Z} \mathbb{L}$ is given by the equation $x^{\sim}=x^{\prime}$. In any BZ-lattice, we set $\diamond x=x^{\sim \sim}$ and $\square x=x^{\prime \sim}$. The following arithmetical lemma, the proof of which is variously scattered in the above-mentioned literature and elsewhere, will be used with no special mention in what follows.

Lemma 2.1. Let $\mathbf{L}$ be a BZ-lattice. For all $a, b \in L$, the following conditions hold: 
(i): $a^{\sim \sim \sim}=a^{\sim}$;

(ii): $a^{\sim} \leq a^{\prime}$;

(iii): $(a \vee b)^{\sim}=a^{\sim} \wedge b^{\sim}$;

(iv): $a^{\sim} \vee b^{\sim} \leq(a \wedge b)^{\sim}$;

(v): $\left(\square\left(a^{\prime}\right)\right)^{\prime}=\diamond a$;

(vi): $\square(a \wedge b)=\square a \wedge \square b$;

(vii): $\diamond(a \vee b)=\diamond a \vee \diamond b$;

(viii): $\diamond(a \wedge b) \leq \diamond a \wedge \diamond b$;

(ix): if $a^{\prime} \leq a$, then $a^{\sim}=0$.

We remarked above that Kleene-sharpness is not the unique purely algebraic characterisation of a sharp quantum property. Two noteworthy alternatives now become available in our expanded language of BZ-lattices.

Definition 2.5. Let $\mathbf{L}$ be a BZ-lattice.

(1) An element $a \in L$ is said to be $\diamond$-sharp iff $a=\diamond a$ (equivalently, iff $a^{\prime}=a^{\sim}$ ); the class of all $\diamond$-sharp elements of $\mathbf{L}$ will be denoted by $S_{\diamond}(L)$.

(2) An element $a \in L$ is said to be Brouwer-sharp iff $a \vee a^{\sim}=1$; the class of all Brouwer-sharp elements of $\mathbf{L}$ will be denoted by $S_{B}(L)$.

For a generic BZ-lattice $\mathbf{L}$, we have that $S_{\diamond}(L) \subset S_{B}(L) \subset S_{K}(L)$. However, in any BZlattice of effects of a Hilbert space (under the meet and join operation induced by the spectral ordering) these three classes coincide. Consequently, it makes sense to investigate whether there is a class of BZ-lattices for which this collapse result can be recovered at a purely abstract level. The next definition and theorem answer this question in the affirmative.

Definition 2.6. A BZ*-lattice is a BZ-lattice $\mathbf{L}$ that satisfies the following condition for all $a \in L:$

$$
\text { (*) } \quad\left(a \wedge a^{\prime}\right)^{\sim} \leq a^{\sim} \vee a^{\prime \sim} .
$$

Theorem 2.1. Let $\mathbf{L}$ be a paraorthomodular BZ*-lattice. Then,

$$
S_{\diamond}(L)=S_{B}(L)=S_{K}(L) .
$$

As pleasing as this result may be, the class of paraorthomodular BZ*-lattices still suffers from a major shortcoming: the paraorthomodularity condition is quasiequational. However, the next result shows that it can be replaced by an equation.

Theorem 2.2. Let $\mathbf{L}$ be a BZ*-lattice. The following conditions are equivalent: 
(1) $\mathbf{L}$ is paraorthomodular;

(2) L satisfies the following $\diamond$-orthomodularity condition for all $a, b \in L$ :

$$
\left(a^{\sim} \vee(\diamond a \wedge \diamond b)\right) \wedge \diamond a \leq \diamond b .
$$

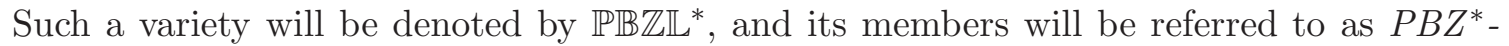
lattices. It can be seen that every bounded lattice can be embedded as a sublattice into

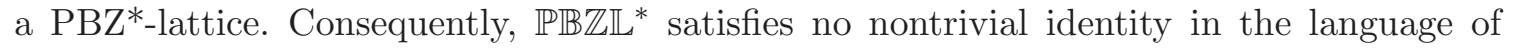
lattices.

The naturalness of this concept is further reinforced by the circumstance that BZ-lattices of effects of a Hilbert space, under the spectral ordering, qualify as instances of PBZ*lattices:

Theorem 2.3. Let $\mathbf{H}$ be a Hilbert space. The algebra

$$
\mathbf{E}(\mathbf{H})=\left\langle\mathcal{E}(\mathbf{H}), \wedge_{s}, \vee_{s}^{\prime},{ }^{\prime} \sim, \mathbb{O}, \mathbb{I}\right\rangle,
$$

(see the introduction for the notation) is a PBZ*-lattice. Moreover, $S_{K}(\mathbf{E}(\mathbf{H}))=S_{\diamond}(\mathbf{E}(\mathbf{H}))=$ $S_{B}(\mathbf{E}(\mathbf{H}))$ is an orthomodular subuniverse of $\mathbf{E}(\mathbf{H})$ consisting of all the projection operators of $\mathbf{H}$.

All orthomodular lattices are, of course, PBZ*-lattices. Further examples of PBZ*-lattices are given by those algebras in this class which are "as far apart as possible" from orthomodular lattices. In any orthomodular lattice $\mathbf{L}, S_{K}(L)=L$; on the other hand, $\mathbf{L}$ is an antiortholattice if $S_{K}(L)=\{0,1\}$. We denote by $\mathbb{A O L}$ the class of antiortholattices. For all $n \geq 1$, the $n$-element Kleene chain $\mathbf{D}_{n}$, such that $a^{\sim}=0$ iff $a>0$, is an antiortholattice.

Some elementary properties of antiortholattices follow.

\section{Lemma 2.2.}

(1) $\mathbf{L} \in \mathbb{A O L}$ iff, for all $a \in L, a^{\sim}=0$ if $a>0$ and $a^{\sim}=1$ otherwise;

(2) Every $\mathbf{L} \in \mathbb{A} \mathbb{O L}$ is directly indecomposable;

(3) $\mathbb{A O L}$ is a proper universal class.

An easy observation that paves the way for the study of the lattice $\mathbf{L}_{\mathbb{P B} \mathbb{Z} \mathbb{L}^{*}}$ of subvarieties

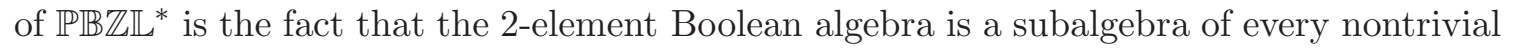

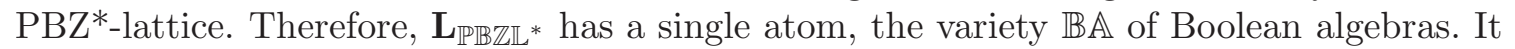
is well-known that $\mathbb{B} \mathbb{A}$ has a single orthomodular cover [3, Cor. 3.6]: the variety $V\left(\mathbf{M O}_{2}\right)$, generated by the simple modular ortholattice with 4 atoms. Two questions naturally arise: how many non-orthomodular covers of the Boolean variety are there? Is it possible to give a finite equational basis for the variety $V(\mathbb{A O L})$ generated by antiortholattices? The next theorem provides a solution to these problems. 
Theorem 2.4. (1) An equational basis for $V(\mathbb{A O L})$ relative to $\mathbb{P B \mathbb { Z }}{ }^{*}$ is given by the identities

$(\mathrm{AOL} 1)\left(x^{\sim} \vee y^{\sim}\right) \wedge\left(\diamond x \vee z^{\sim}\right) \approx\left(\left(x^{\sim} \vee y\right) \wedge(\diamond x \vee z)\right)^{\sim}$

$(\mathrm{AOL} 2) x \approx\left(x \wedge y^{\sim}\right) \vee(x \wedge \diamond y)$

$\left(\right.$ AOL3) $x \approx\left(x \vee y^{\sim}\right) \wedge(x \vee \diamond y)$.

(2) $\mathbb{O M L} \cap V(\mathbb{A O L})=\mathbb{B A}$;

(3) There is a single non-orthomodular cover of $\mathbb{B} \mathbb{A}$, the variety $V\left(\mathbf{D}_{3}\right)$ generated by the 3-element antiortholattice chain.

We recall from [11] that $V\left(\mathbf{D}_{3}\right)$ is axiomatised relative to $V(\mathbb{A O L})$ by the identities

$$
\begin{aligned}
(\mathrm{DIST}) x \wedge(y \vee z) & \approx(x \wedge y) \vee(x \wedge z) ; \\
(\mathrm{SDM})(x \wedge y)^{\sim} & \approx x^{\sim} \vee y^{\sim} ; \\
(\mathrm{SK}) x \wedge \diamond y & \leq \square x \vee y .
\end{aligned}
$$

\section{More on the LAttice of PBZ* VARieties}

In this section, we intend to explore in greater detail the structure of $\mathbf{L}_{\mathbb{P B Z} \mathbb{L}^{*} \text {, extending }}$ the preliminary results on its structure contained in [12]. In particular, we will focus on the antiortholattice side of $\mathbf{L}_{\mathbb{B} \mathbb{Z} L^{*}}$ and try to investigate some properties of $V(\mathbb{A O L})$ and some of its notable subvarieties. The only exception to this policy is the next lemma, which disproves the natural conjecture to the effect that the join of the orthomodular variety $\mathbb{O M L}$ and the antiortholattice variety $V(\mathbb{A O L})$ covers the whole of $\mathbb{P B Z \mathbb { L }}$.

Lemma 3.1. $V(\mathbb{A O L}) \vee \mathbb{O M L}<\mathbb{P B Z L}$.

Proof. The identity

$$
x \vee y \approx\left((x \vee y) \wedge y^{\sim}\right) \vee((x \vee y) \wedge \diamond y)
$$

is readily seen to hold both in $\mathbb{A O L}$ and in $\mathbb{O M L}$. However, it fails in $\mathbb{P B Z L}{ }^{*}$. In fact, consider the $\mathrm{PBZ}^{*}$-lattice displayed in the next figure:

\section{[Missing Picture]}

with $a^{\sim}=b^{\prime \sim}=b, b^{\sim}=b^{\prime}$ and $a^{\prime \sim}=c^{\sim}=0$. Our conclusion follows upon observing that

$$
c \vee a=c \neq a=\left((c \vee a) \wedge a^{\sim}\right) \vee((c \vee a) \wedge \diamond a) .
$$


Let us now comfortably settle within the boundaries of $V(\mathbb{A O L})$. For a start, consider the next three varieties:

$$
\begin{aligned}
\mathbb{V}_{1} & =\operatorname{Mod}(E q(\mathbb{A O L}) \cup\{\mathrm{DIST}\}) \\
\mathbb{V}_{2} & =\operatorname{Mod}(E q(\mathbb{A O L}) \cup\{\mathrm{SDM}\}) ; \\
\mathbb{V}_{3} & =\operatorname{Mod}(E q(\mathbb{A O L}) \cup\{\mathrm{DIST}, \mathrm{SDM}\}) .
\end{aligned}
$$

Lemma 3.2. (i) $V\left(\mathbf{D}_{3}\right)<\mathbb{V}_{3}$; (ii) $\mathbb{V}_{1}$ and $\mathbb{V}_{2}$ are incomparable varieties, hence strictly smaller than $V(\mathbb{A O L})$.

Proof. (i) It is easy to check that the algebra $\mathbf{D}_{4}$ belongs to $\mathbb{V}_{3}$. Now, let $a, a^{\prime}$ denote the elements of $D_{4}$ different from the bounds, s.t. $a<a^{\prime}$. We have that $a^{\prime}=a^{\prime} \wedge \diamond a>\square a^{\prime} \vee a=$ $a$, whence $\mathbf{D}_{4}$ fails $\mathrm{SK}$.

(ii) The antiortholattice

\section{[Missing Picture]}

is a non-distributive algebra in $\mathbb{V}_{2}$. The next distributive antiortholattice:

$$
\text { [Missing Picture] }
$$

fails SDM because $(a \wedge b)^{\sim}=0^{\sim}=1>0=0 \vee 0=a^{\sim} \vee b^{\sim}$.

3.1. The variety generated by antiortholattices. Next, we prove a representation theorem for the subdirectly irreducible algebras in $V(\mathbb{A O L})$. We intend to put to good use a certain kind of twist construction, along the lines of what is done in $[16,8,6,12]$ and in several other papers on pseudo-Kleene algebras and related structures.

Let $\mathbf{L}$ be a PBZ*-lattice. We define:

$$
\begin{aligned}
N(\mathbf{L}) & =\left\{a \in L: a \leq a^{\prime}\right\} \quad \text { (the set of negative elements); } \\
P(\mathbf{L}) & =\left\{a \in L: a^{\prime} \leq a\right\} \quad \text { (the set of positive elements); } \\
N(\mathbf{L})^{+} & =\left\{a \in L: a<a^{\prime}\right\} \quad \text { (the set of strictly negative elements); } \\
P(\mathbf{L})^{+} & =\left\{a \in L: a^{\prime}<a\right\} \quad \text { (the set of strictly positive elements); }
\end{aligned}
$$

By the pseudo-Kleene quasiequation, $N(\mathbf{L})$ and $P(\mathbf{L})$ are subuniverses of the lattice reduct of $\mathbf{L}$. With a slight notational abuse, we continue to denote by the same symbols $N(\mathbf{L})$ and $P(\mathbf{L})$, respectively, the corresponding lattice subreducts of $\mathbf{L}$.

Lemma 3.3. Let $\mathbf{L} \in V(\mathbb{A O L})$ be a subdirectly irreducible algebra. Then:

(1) $\mathbf{L}$ is an antiortholattice;

(2) $P(\mathbf{L}) \cup N(\mathbf{L})=L$. 
Proof. (1) If $\mathbf{L}$ is s.i., then it is directly indecomposable, whence its central elements are $0,1$. Since $\mathbf{L}$ is in $V(\mathbb{A O L})$, its central elements coincide with its sharp elements. So $S_{K}(\mathbf{L})=$ $\{0,1\}$ and $\mathbf{L}$ is an antiortholattice.

(2) By (1), we can safely assume that $\mathbf{L}$ is an antiortholattice. Suppose ex absurdo that there exists $a$ such that $a \not \leq a^{\prime}$ and $a^{\prime} \not \leq a$. Let $\theta_{1}$ be the equivalence whose unique non-singleton classes are the intervals $\left[a \wedge a^{\prime}, a\right]$ and $\left[a^{\prime}, a \vee a^{\prime}\right]$; likewise, let $\theta_{2}$ be the equivalence whose unique non-singleton classes are the intervals $\left[a \wedge a^{\prime}, a^{\prime}\right]$ and $\left[a, a \vee a^{\prime}\right]$. Indeed, we have that $\theta_{1}, \theta_{2}>\Delta$ : in fact, if $\left[a \wedge a^{\prime}, a\right]$ were a singleton, then $a \leq a^{\prime}$, against our hypothesis, and similarly for the remaining intervals.

We now show that $\theta_{1}$ is a congruence. Disregarding trivial cases, if $\left(b_{1}, c_{1}\right),\left(b_{2}, c_{2}\right) \in \theta_{1}$, then we have to distinguish different situations. If $a \wedge a^{\prime} \leq b_{1}, c_{1}, b_{2}, c_{2} \leq a$, then clearly

$$
a \wedge a^{\prime} \leq b_{1} \wedge b_{2}, c_{1} \wedge c_{2} \leq a
$$

and thus $\left(b_{1} \wedge b_{2}, c_{1} \wedge c_{2}\right) \in \theta_{1}$. If $a \wedge a^{\prime} \leq b_{1}, c_{1} \leq a$ and $a^{\prime} \leq b_{2}, c_{2} \leq a \vee a^{\prime}$, then $a \wedge a^{\prime} \leq a^{\prime} \leq b_{2}, c_{2}$, and thus

$$
a \wedge a^{\prime} \leq b_{1} \wedge b_{2} \leq b_{1} \leq a \text { and } a \wedge a^{\prime} \leq c_{1} \wedge c_{2} \leq c_{1} \leq a
$$

and the same conclusion follows. The other cases are disposed of similarly, whence $\theta_{1}$ preserves meets. It is readily seen that if $b, c \in\left[a \wedge a^{\prime}, a\right]$, then $b^{\prime}, c^{\prime} \in\left[a^{\prime}, a \vee a^{\prime}\right]$, and vice versa. Finally, our hypothesis that $a \not \leq a^{\prime}$ and $a^{\prime} \not \leq a$ implies that $0<a, a^{\prime}$; if it were that $a \wedge a^{\prime}=0$, then $a$ would be a nonzero sharp element, against the fact that $\mathbf{L}$ is an antiortholattice. In sum, if either $b, c \in\left[a \wedge a^{\prime}, a\right]$ or $b, c \in\left[a^{\prime}, a \vee a^{\prime}\right]$, then $0<b, c$ and then $b^{\sim}=c^{\sim}=0$. Similarly, it can be shown that $\theta_{2}$ is a congruence.

To obtain a contradiction, it remains to be proved that $\theta_{1} \cap \theta_{2}=\Delta$. Again, we have to split cases. If $a \wedge a^{\prime} \leq b, c \leq a$ and $a \wedge a^{\prime} \leq b, c \leq a^{\prime}$, then $a \wedge a^{\prime} \leq b, c \leq a \wedge a^{\prime}$, whence $b=a \wedge a^{\prime}=c$. If $a \wedge a^{\prime} \leq b, c \leq a$ and $a \leq b, c \leq a \vee a^{\prime}$, then

$$
a \wedge a^{\prime} \leq b, c \leq a \leq b, c,
$$

whereby, again, $b=a=c$. The remaining cases yield similar outcomes.

We now introduce a slight variant of the twist construction already used in [12]. Let $\mathbf{L}=\langle L, \wedge, \vee\rangle$ be a bounded lattice with bounds 0 and 1 and induced order $\leq$. Take a bijective copy $f[L-\{0\}]$ of $L-\{0\}$ and endow it with the order $\leq^{\partial}$ dual to $\leq$; call the resulting lattice $\mathbf{M}$. Now, consider the ordinal sum $\mathbf{M} \oplus \mathbf{L}[15$, Ch. 4], which is a lattice. Let, for $a \in M \oplus L$,

$$
a^{\prime}=\left\{\begin{array}{l}
f(a) \text { if } a \in L-\{0\} \\
0 \text { if } a=0 \\
f^{-1}(a) \text { if } a \in M
\end{array}\right.
$$

The algebra $\mathcal{T}_{1}(\mathbf{L})=\left\langle M \oplus L, \wedge^{\mathcal{T}_{1}(\mathbf{L})}, \vee^{\mathcal{T}_{1}(\mathbf{L})}, \mathcal{T}(\mathbf{L}), \sim \mathcal{T}_{1}(\mathbf{L}), 0^{\mathcal{T}_{1}(\mathbf{L})}, 1^{\mathcal{T}_{1}(\mathbf{L})}\right\rangle$, where:

$$
\begin{aligned}
& \wedge^{\mathcal{T}_{1}(\mathbf{L})}=\wedge^{\mathbf{M} \oplus \mathbf{L}} ; \quad \vee^{\mathcal{T}_{1}(\mathbf{L})}=\vee^{\mathbf{M} \oplus \mathbf{L}} ; \quad 0^{\mathcal{T}_{1}(\mathbf{L})}=f(1) ; \\
& { }^{\prime \mathcal{T}_{1}(\mathbf{L})}={ }^{\prime} ; \quad 1^{\mathcal{T}_{1}(\mathbf{L})}=1
\end{aligned}
$$


and

$$
a^{\sim \mathcal{T}_{1}(\mathbf{L})}=\left\{\begin{array}{l}
1 \text { if } a=0^{\mathcal{T}_{1}(\mathbf{L})} \\
0^{\mathcal{T}_{1}(\mathbf{L})} \text { otherwise }
\end{array}\right.
$$

is an antiortholattice such that $P\left(\mathcal{T}_{1}(\mathbf{L})\right) \cup N\left(\mathcal{T}_{1}(\mathbf{L})\right)=M \oplus L$, and $\mathbf{L}$ is a lattice subreduct of such - actually, $L=P\left(\mathcal{T}_{1}(\mathbf{L})\right)$. A similar construction can be effected for any upper bounded lattice $\mathbf{L}$, with the only difference that we take a bijective copy $f[L]$ of the whole universe $L$ of the lattice at issue, not just of $L-\{0\}$. The resulting PBZ*-lattice, which we call $\mathcal{T}_{2}(\mathbf{L})$, will be such that:

- if $\mathbf{L}$ has a bottom element 0 , then $P\left(\mathcal{T}_{2}(\mathbf{L})\right)$ has 0 as a bottom element and $N\left(\mathcal{T}_{2}(\mathbf{L})\right)$ has $f(0)$ as a top element;

- if $\mathbf{L}$ is unbounded below, then $P\left(\mathcal{T}_{2}(\mathbf{L})\right)$ has no bottom element and $N\left(\mathcal{T}_{2}(\mathbf{L})\right)$ has no top element.

Conversely, we have that:

Theorem 3.1. Every nontrivial antiortholattice $\mathbf{L}$ such that $P(\mathbf{L}) \cup N(\mathbf{L})=L$ is isomorphic to $\mathcal{T}_{i}(P(\mathbf{L}))$, for some $i \in\{1,2\}$.

Proof. Let $i$ be 1 or 2 according as there is $c \in L$ such that $c=c^{\prime}$ or not. In the former case, in fact, $c$ is the bottom element in $P(\mathbf{L})$ and the construction can be applied. Let $\varphi: L \rightarrow \mathcal{T}_{i}(P(\mathbf{L}))$ be defined by:

$$
\varphi(a)=\left\{\begin{array}{l}
a \text { if } a \in P(\mathbf{L}) \\
f\left(a^{\prime \mathbf{L}}\right) \text { otherwise }
\end{array}\right.
$$

This function is clearly injective, and it is surjective because $P(\mathbf{L}) \cup N(\mathbf{L})=L$ and $f$ is a bijection. Moreover, $\varphi\left(0^{\mathbf{L}}\right)=f\left(1^{\mathbf{L}}\right)=0^{\mathcal{T}_{i}(\mathbf{L})}$.

Let us check that $\varphi\left(a \wedge^{\mathbf{L}} b\right)=\varphi(a) \wedge^{\mathcal{T}_{i}(P(\mathbf{L}))} \varphi(b)$. If $a \wedge^{\mathbf{L}} b \in P(\mathbf{L})$, then $a, b \in P(\mathbf{L})$, and our conclusion is trivial. On the other hand, if $a \wedge^{\mathbf{L}} b \in N(\mathbf{L})^{+}$, then suppose further that $a, b \in N(\mathbf{L})^{+}$. Then

$$
\begin{aligned}
\varphi\left(a \wedge^{\mathbf{L}} b\right) & =f\left(\left(a \wedge^{\mathbf{L}} b\right)^{\prime \mathbf{L}}\right) \\
& =f\left(a^{\mathbf{L}} \vee^{\mathbf{L}} b^{\prime \mathbf{L}}\right) \\
& =f\left(a^{\prime \mathbf{L}}\right) \wedge^{\mathcal{T}_{i}(\mathbf{L})} f\left(b^{\prime \mathbf{L}}\right) \\
& =\varphi(a) \wedge^{\mathcal{T}_{i}(P(\mathbf{L}))} \varphi(b) .
\end{aligned}
$$

Suppose instead w.l.g. that $a \in N(\mathbf{L})^{+}, b \in P(\mathbf{L})$. Then

$$
\varphi\left(a \wedge^{\mathbf{L}} b\right)=\varphi(a)=f\left(a^{\prime \mathbf{L}}\right)=f\left(a^{\prime \mathbf{L}}\right) \wedge^{\mathcal{T}_{i}(\mathbf{L})} b=\varphi(a) \wedge^{\mathcal{T}_{i}(P(\mathbf{L}))} \varphi(b) .
$$

Next, we verify that $\varphi\left(a^{\prime \mathbf{L}}\right)=\varphi(a)^{\prime \mathcal{T}_{i}(\mathbf{L})}$. If $a \in N(\mathbf{L})^{+}$, then $a^{\prime \mathbf{L}} \in P(\mathbf{L})$ and so $\varphi\left(a^{\prime \mathbf{L}}\right)=$ $a^{\prime \mathbf{L}}=f^{-1}\left(f\left(a^{\prime \mathbf{L}}\right)\right)=f^{-1}(\varphi(a))=\varphi(a)^{\prime \mathcal{T}_{i}(\mathbf{L})}$. If $a \in P(\mathbf{L})^{+}$, then $a^{\prime \mathbf{L}} \in N(\mathbf{L})^{+}$and so 
$\varphi\left(a^{\prime \mathbf{L}}\right)=f(a)=f(\varphi(a))=\varphi(a)^{\prime \mathcal{T}_{i}(\mathbf{L})}$. If $a=c=c^{\prime}$, then $\varphi\left(a^{\prime \mathbf{L}}\right)=\varphi(c)=c=c^{\prime T_{1}(\mathbf{L})}=$ $\varphi(a)^{\prime \mathcal{T}_{1}(\mathbf{L})}$.

Finally, we check that $\varphi\left(a^{\sim \mathbf{L}}\right)=\varphi(a)^{\sim \mathcal{T}_{i}(\mathbf{L})}$. If $a=0^{\mathbf{L}}$, then $\varphi\left(a^{\sim \mathbf{L}}\right)=1^{\mathbf{L}}=f(1)^{\sim \mathcal{T}_{i}(\mathbf{L})}=$ $\varphi(a)^{\sim \mathcal{T}_{i}(\mathbf{L})}$. If $a>0^{\mathbf{L}}$, then $\varphi\left(a^{\sim \mathbf{L}}\right)=\varphi\left(0^{\mathbf{L}}\right)=f(1)$. Moreover, $f(1)=a^{\sim \mathcal{T}_{i}(\mathbf{L})}=$ $\varphi(a)^{\sim \mathcal{T}_{i}(\mathbf{L})}$ if $a \in P(\mathbf{L})$ and, since $a^{\prime}<1^{\mathbf{L}}, f(1)=\left(f\left(a^{\prime \mathbf{L}}\right)\right)^{\sim \mathcal{T}_{i}(\mathbf{L})}=\varphi(a)^{\sim \mathcal{T}_{i}(\mathbf{L})}$ if $a \in$ $N(\mathbf{L})^{+}$.

From Lemma 3.3 and Theorem 3.1 the following representation of subdirectly irreducible members of $V(\mathbb{A O L})$ immediately follows:

Corollary 3.1. If $\mathbf{L} \in V(\mathbb{A O L})$ is a subdirectly irreducible algebra, then $\mathbf{L}$ is isomorphic to $\mathcal{T}_{i}(P(\mathbf{L}))$, for some $i \in\{1,2\}$.

3.2. The distributive subvariety. In this very short subsection we make a note of a small observation about the distributive subvariety $\mathbb{V}_{1}$ of $V(\mathbb{A O L})$, parlaying Corollary 3.1 above into a characterisation of a notable set of generators for $\mathbb{V}_{1}$. The intrinsic interest of this distributive subvariety is further reinforced by recalling that the ${ }^{\sim}$-free reducts of its members are Kleene lattices, whence they belong to the variety of pseudo-Kleene algebras generated by the ${ }^{\sim}$-free reduct of $\mathbf{D}_{3}$.

Hereafter, by $\mathbf{2}$ we denote the 2 -element chain, considered as a lattice.

Theorem 3.2. $\mathbb{V}_{1}$ is generated by the class $\left\{\mathcal{T}_{i}\left(\mathbf{2}^{\kappa}\right): i \in\{1,2\}, \kappa\right.$ a cardinal $\}$.

Proof. Let $\mathbf{L} \in \mathbb{V}_{1}$ be a subdirectly irreducible algebra. By Corollary 3.1, $\mathbf{L}$ is isomorphic to $\mathcal{T}_{i}(\mathbf{M})$, for some $i \in\{1,2\}$ and for some distributive lattice $\mathbf{M}$ (since the twist product construction is easily seen to preserve distributivity). Since the class of distributive lattices is generated as a quasivariety by the single finite algebra $\mathbf{2}$, it follows that $\mathbf{L} \in I \mathcal{T}_{i}(\operatorname{ISP}(\mathbf{2}))$. However, it is readily observed that the mappings $\mathcal{T}_{i}$, viewed as operators that map classes of lattices to classes of antiortholattices, commute with the class operators $I$ and $S$ (in their respective signatures). Therefore $\mathbf{L} \in I S \mathcal{T}_{i}(P(\mathbf{2}))$ and thus $\mathbb{V}_{1}$ si $\subseteq I S \mathcal{T}_{i}(P(\mathbf{2}))$, whence $\mathbb{V}_{1}=V\left(\mathbb{V}_{1} s i\right) \subseteq H S P I S \mathcal{T}_{i}(P(\mathbf{2})) \subseteq V\left(\mathcal{T}_{i}(P(\mathbf{2}))\right)=V\left(\left\{\mathcal{T}_{i}\left(\mathbf{2}^{\kappa}\right): i \in\{1,2\}, \kappa\right.\right.$ a cardinal $\left.\}\right)$.

Conversely, it is clear that every member of $\left\{\mathcal{T}_{i}\left(\mathbf{2}^{\kappa}\right): i \in\{1,2\}, \kappa\right.$ a cardinal $\}$ is a distributive antiortholattice.

3.3. The distributive subvariety with the strong De Morgan property. The main result of this subsection is a theorem to the effect that $\mathbb{V}_{3}$, the distributive subvariety of $V(\mathbb{A O L})$ with the strong De Morgan property, is generated by the single 5-element chain $\mathbf{D}_{5}$. We will achieve this result via a variant of the well-known technique deployed by Kalman in [16] in order to show that the 3 -element Kleene chain generates the variety of Kleene lattices. 
For a start, we show that:

Lemma 3.4. Every PBZ* chain is in $\mathbb{V}_{3}$.

Proof. It will suffice to prove that every $\mathrm{PBZ}^{*}$ chain $\mathbf{L}$ is an antiortholattice and satisfies SDM. As to the former claim, suppose $a \in L$. Either $0=a \wedge a^{\sim}=a$ or $0=a \wedge a^{\sim}=a^{\sim}$, which proves our conclusion. For SDM, $(a \wedge 0)^{\sim}=1=a^{\sim} \vee 1=a^{\sim} \vee 0^{\sim}$ for all $a \in A$, so w.l.g. let $a, b \in L$ be such that $a \wedge b>0$. Since $L$ is a linearly ordered antiortholattice, $(a \wedge b)^{\sim}=0=a^{\sim} \vee b^{\sim}$.

We now want to find a suitable set of generators for $\mathbb{V}_{3}$. Let $\mathbf{L} \in \mathbb{V}_{3}$, and let, for any $p \in L$,

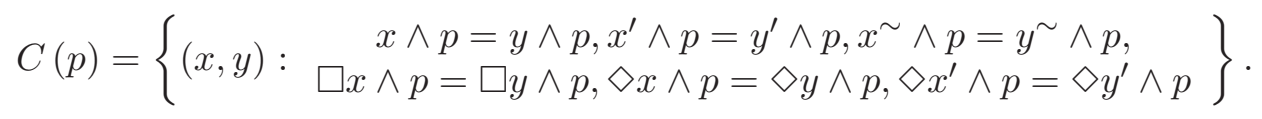

Although this definition looks complicated, observe that if $\mathbf{L} \in \mathbb{V}_{3}$ is subdirectly irreducible (hence an antiortholattice by Lemma 3.3), all of the conditions it contains except for the first two ones trivialise for all pairs $(x, y)$ s.t. $0<x, y<1$.

Lemma 3.5. Let $\mathbf{L} \in \mathbb{V}_{3}$ and let $p, q \in L$. Then: (i) $C(p)$ is a congruence on $\mathbf{L}$; (ii) $C(p) \cap C(q)=C(p \vee q)$; (iii) $C(p) \cap C\left(p^{\prime}\right)=\Delta$; (iv) $C(p)=\Delta$ iff $p \in P(\mathbf{L})$.

Proof. (i), (ii): left to the reader.

(iii). Suppose $(x, y) \in C(p) \cap C\left(p^{\prime}\right)$. Then in particular $x \wedge p=y \wedge p$ and $x^{\prime} \wedge p^{\prime}=y^{\prime} \wedge p^{\prime}$, whence $x \vee p=y \vee p$. By [2, L. 1.(ii), p. 28], $x=y$.

(iv) By (iii), it suffices to prove that $C(p) \subseteq C\left(p^{\prime}\right)$ iff $p^{\prime} \leq p$. From left to right, it can be checked that $\left(p \wedge p^{\prime}, p^{\prime}\right) \in C(p) \subseteq C\left(p^{\prime}\right)$, whence $p \wedge p^{\prime}=p^{\prime}$. Conversely, suppose $p^{\prime} \leq p$ and $(x, y) \in C(p)$. Since $x \wedge p=y \wedge p$, we have that

$$
x \wedge p^{\prime}=x \wedge p^{\prime} \wedge p=y \wedge p^{\prime} \wedge p=y \wedge p^{\prime},
$$

and similarly for the other conditions.

Lemma 3.6. Let $\mathbf{L} \in \mathbb{V}_{3}$ be a subdirectly irreducible algebra, and let $a, b \in L$. Then: (i) $P(\mathbf{L})^{+}$is closed under meets; (ii) if $a \in P(\mathbf{L})^{+}, b \in N(\mathbf{L})$, then $b<a$.

Proof. (i) Suppose $a^{\prime}<a, b^{\prime}<b$, but $a^{\prime} \vee b^{\prime} \nless a \wedge b$. By Lemma 3.3.(ii), $a \wedge b \leq a^{\prime} \vee b^{\prime}$, and thus by Lemma 3.5.(ii)-(iv) $\Delta=C\left(a^{\prime} \vee b^{\prime}\right)=C\left(a^{\prime}\right) \cap C\left(b^{\prime}\right)$. Thus, $\Delta=C\left(a^{\prime}\right)$ or $\Delta=C\left(b^{\prime}\right)$, whence using Lemma 3.5.(iv) again, either $a \leq a^{\prime}$ or $b \leq b^{\prime}$, a contradiction.

(ii) Suppose $a^{\prime}<a, b \leq b^{\prime}$. By Lemma 3.3.(ii), either $a \wedge b^{\prime}<a^{\prime} \vee b$ or $a^{\prime} \vee b \leq a \wedge b^{\prime}$. If $a \wedge b^{\prime}<a^{\prime} \vee b$, then by the previous item

$$
a \wedge\left(a^{\prime} \vee b\right) \leq a \wedge\left(a^{\prime} \vee b^{\prime}\right)=a^{\prime} \vee\left(a \wedge b^{\prime}\right)<a \wedge\left(a^{\prime} \vee b\right)
$$


a contradiction. So $a^{\prime} \vee b \leq a \wedge b^{\prime}$, whence $b \leq a^{\prime} \vee b \leq a \wedge b^{\prime} \leq a$. But $a \neq b$, since otherwise $b \leq b^{\prime}<b$. Our conclusion follows.

Let $\mathbf{L} \in \mathbb{V}_{3}$ be a subdirectly irreducible algebra. Let $\sim$ be the equivalence whose cosets are $\{0\},\{1\}, N(\mathbf{L})^{+}, P(\mathbf{L})^{+}$and the singleton of the fixpoint $c$ (if present). Let moreover, for $p \in L:$

$$
\begin{aligned}
& D(p)=\left\{(x, y): x \sim y \text { and }\left(x \vee x^{\prime}\right) \wedge p=\left(y \vee y^{\prime}\right) \wedge p\right\} \\
& E(p)=\left\{(x, y): x \sim y \text { and }\left(x \vee x^{\prime}\right) \vee p=\left(y \vee y^{\prime}\right) \vee p\right\} .
\end{aligned}
$$

Proposition 3.1. (i) is a congruence; (ii) for all $p \in L, D(p)$ is a congruence; (iii) for all $p \in L, E(p)$ is a congruence; (iv) for all $p \in L$, either $D(p)=\Delta$ or $E(p)=\Delta$.

Proof. (i) preserves meets and Kleene complements by Lemma 3.6. Moreover, the Brouwer complement is preserved because $\mathbf{L}$, by Lemma 3.3.(i), is an antiortholattice.

(ii) It is not hard to show, following in the footsteps of [16], that $D(p)$ preserves meets and Kleene complements; thus, it suffices to show that Brouwer complements are also preserved. If $a \sim b$, either $a=b=0$ or $a, b>0$. We have to prove that

$$
\left(a^{\sim} \vee \diamond a\right) \wedge p=\left(b^{\sim} \vee \diamond b\right) \wedge p
$$

but in both cases the left-hand and the right-hand side reduce to $p$ because $\mathbf{L}$ is an antiortholattice.

(iii) Similar.

(iv) Suppose $(x, y) \in D(p) \cap E(p)$. Then $\left(x \vee x^{\prime}\right) \wedge p=\left(y \vee y^{\prime}\right) \wedge p$ and $\left(x \vee x^{\prime}\right) \vee p=$ $\left(y \vee y^{\prime}\right) \vee p$. By virtue of [2, L. 1.(ii), p. 28], $x \vee x^{\prime}=y \vee y^{\prime}$ and so, since $x \sim y$, we have that $x=y$. Thus $D(p) \cap E(p)=\Delta$, whence our conclusion follows since $\mathbf{L}$ is s.i.

Theorem 3.3. The only nontrivial subdirectly irreducible members of $\mathbb{V}_{3}$ are $\mathbf{D}_{2}, \mathbf{D}_{3}, \mathbf{D}_{4}, \mathbf{D}_{5}$.

Proof. Let $\mathbf{L} \in \mathbb{V}_{3}$ be a nontrivial subdirectly irreducible algebra. So $0<1$. If $L$ contains no strictly positive element $c$, either $\mathbf{L}=\mathbf{D}_{2}$ or $\mathbf{L}=\mathbf{D}_{3}$. Otherwise, we show that there cannot be three distinct such elements. In fact, suppose the contrary. Then these elements must form a chain $0<w<y<x<1$, with $w^{\prime}<w, y^{\prime}<y, x^{\prime}<x$. But it is easy to check that $(x, y) \in D(y)$ and $(w, y) \in E(y)$, contra Proposition 3.1.(iv). So $L$ has at most two strictly positive elements $x, y$, which must be comparable if they exist, and no element $a$ which is not comparable with $a^{\prime}$ by Lemma 3.3.(ii). If

$$
0<x^{\prime}<y^{\prime}<y<x<1,
$$

then

$$
\left\{\{0\},\{1\},\{x\},\left\{x^{\prime}\right\},\left\{y, y^{\prime}\right\}\right\}
$$

is a partition of $L$, and it is easily checked that the corresponding equivalence $F$ is a nonzero congruence (since one of its cosets is not a singleton). Also, $D(y) \neq \Delta$, because $(x, y) \in D(y)$. However, $D(y)$ does not identify $y$ and $y^{\prime}$, whence $F \cap D(y)=\Delta$. This 
contradicts the subdirect irreducibility of $\mathbf{L}$. So $L$ contains a chain $0<c<c^{\prime}<1$, while it may or may not contain a fixpoint $d=d^{\prime}$. If so, $\mathbf{L}=\mathbf{D}_{5}$; if not, $\mathbf{L}=\mathbf{D}_{4}$.

Corollary 3.2. $\mathbb{V}_{3}=V\left(\mathbf{D}_{5}\right)$.

Proof. In fact, $\mathbf{D}_{i} \leq \mathbf{D}_{5}$ for $i \leq 5$.

3.4. A better basis for $V\left(\mathbf{D}_{3}\right)$. Finally, we give a result for the single non-orthomodular cover of the Boolean variety in $\mathbf{L}_{\mathbb{P B} \mathbb{Z}} \mathbb{L}^{*}$, namely, the variety $V\left(\mathbf{D}_{3}\right)$ generated by the 3element chain $\mathbf{D}_{3}$. As we already recalled, one of the present writers proved in [11] that $V\left(\mathbf{D}_{3}\right)$ is axiomatised relative to $V(\mathbb{A O L})$ by the identities DIST, SDM and SK. The aim of this subsection is showing that this basis is redundant, because

$$
V\left(\mathbf{D}_{3}\right)=\operatorname{Mod}(E q(\mathbb{A O L}) \cup\{\mathrm{SK}\}) .
$$

In other words, SK implies both DIST and SDM in the presence of the antiortholattice axioms.

Lemma 3.7. Let $\mathbf{L} \in \operatorname{Mod}(E q(\mathbb{A O L}) \cup\{\mathrm{SK}, \mathrm{SDM}\})$. Then, for any $a, b, c \in L:$ (i) $a \vee$ $\square b=(a \vee b) \wedge(\diamond a \vee \square b)$; (ii) $a \vee(b \wedge c)=a \vee((\diamond b \vee \square a) \wedge(a \vee b) \wedge c)$; (iii) $a \vee(b \wedge c)=$ $a \vee((a \vee b) \wedge c)=;($ iv $) a \wedge(b \vee c)=a \wedge(b \vee(a \wedge c)) ;(\mathrm{v}) a \wedge(b \vee c)=(a \wedge b) \vee(a \wedge c)$

Proof. (i)

$$
\begin{aligned}
a \vee \square b & =a \vee \square b \vee(\diamond a \wedge b) & & (\mathrm{SK}) \\
& =a \vee((\square b \vee \diamond a) \wedge b) & & {[12, \text { L. 4.1, Prop. 5.2] }} \\
& =(a \vee b) \wedge(a \vee \square b \vee \diamond a) & & {[12, \text { L. 5.10.(ix)], SDM }} \\
& =(a \vee b) \wedge(\diamond a \vee \square b) & & \text { Def. 2.4 }
\end{aligned}
$$

(ii)

$$
\begin{aligned}
a \vee((\diamond b \vee \square a) \wedge(a \vee b) \wedge c) & =a \vee((\square a \vee b) \wedge c) & & \text { (i) } \\
& =a \vee(\square a \wedge c) \vee(b \wedge c) & & {[12, \text { L. 5.10.(ix })] } \\
& =((a \vee \square a) \wedge(a \vee c)) \vee(b \wedge c) & & {[12, \text { L. 5.10.(ix })] } \\
& =a \vee(b \wedge c) & & \text { [12, L. 4.1], Abs. }
\end{aligned}
$$

(iii)

$$
\begin{aligned}
a \vee(b \wedge c) & =a \vee((\diamond b \vee \square a) \wedge c \wedge(a \vee b)) & & \text { (ii) } \\
& =(a \vee \diamond b \vee \square a) \wedge(a \vee(c \wedge(a \vee b))) & & {[12, \text { L. 5.10.(ix })], \text { SDM } } \\
& =a \vee((a \vee b) \wedge c) & & {[12, \text { L. 4.1] }}
\end{aligned}
$$

(iv) From (iii) by duality.

( $\mathrm{v})$

$$
\begin{aligned}
a \wedge(b \vee c) & =a \wedge(c \vee(a \wedge b)) & & \text { (iv) } \\
& =(a \wedge b) \vee(a \wedge(c \vee(a \wedge b))) & & \text { Lattice prop. } \\
& =(a \wedge b) \vee(a \wedge c) & & \text { (iii) }
\end{aligned}
$$


Thus, DIST follows once SDM is satisfied. The next step consists in showing that SDM directly follows from SK in the presence of the remaining axioms of $V(\mathbb{A O L})$.

Lemma 3.8. Let $\mathbf{L} \in \operatorname{Mod}(E q(\mathbb{A O L}) \cup\{\mathrm{SK}\})$. Then, for any $a, b \in L$ : (i) if $a \wedge b=0$, then either $a=0$ or $b=0$; (ii) $(a \wedge b)^{\sim}=a^{\sim} \vee b^{\sim}$.

Proof. (i) L is a subdirect product of antiortholattices in $\operatorname{Mod}(E q(\mathbb{A O L}) \cup\{\mathrm{SK}\})$ by Lemma 3.3.(i), whence it belongs to the quasivariety generated by such antiortholattices. Now, suppose ex absurdo that $a \wedge b=0$, but $a, b>0$. This immediately implies that $a, b<1$. By SK, moreover, we would have that $a=a \wedge \diamond b \leq \square a \vee b=b$, whence $a \wedge b=a>0$, a contradiction.

(ii) By Lemma 3.3.(i), we can safely assume that $\mathbf{L}$ is an antiortholattice. If $a \wedge b>0$, then $a, b>0$, whence $(a \wedge b)^{\sim}=0=a^{\sim} \vee b^{\sim}$. If $a \wedge b=0$, then by (i) either $a=0$ or $b=0$, and thus $(a \wedge b)^{\sim}=1=a^{\sim} \vee b^{\sim}$.

Corollary 3.3. $V\left(\mathbf{D}_{3}\right)=\operatorname{Mod}(E q(\mathbb{A O L}) \cup\{\mathrm{SK}\})$.

Proof. Our result follows from Lemmas 3.7 and 3.8.

\section{Horizontal SUMS}

The technique of horizontal sums, in itself a crucial tool for lattice theorists, has been investigated for orthomodular lattices e.g. in [7, 13]. In [12], we adapted it to PBZ*-lattices along the following lines.

Let $\mathbf{L}$ be an orthomodular lattice and $\mathbf{M}$ be any PBZ*-lattice, with $L \cap M=\{0,1\}$. The horizontal sum $\mathbf{L} \boxplus \mathbf{M}$ of $\mathbf{L}, \mathbf{M}$ is the algebra

$$
\left\langle L \cup M, \wedge^{\mathbf{L} \boxplus \mathbf{M}}, \vee^{\mathbf{L} \boxplus \mathbf{M}}, / \mathbf{L} \boxplus \mathbf{M}, \sim \mathbf{L} \boxplus \mathbf{M}, 0,1\right\rangle,
$$

where for $a \in L, a^{\prime \mathbf{L} \boxplus \mathbf{M}}=a^{\prime \mathbf{L}}, a^{\sim \mathbf{L} \boxplus \mathbf{M}}=a^{\sim \mathbf{L}}$, while for $a \in M, a^{\prime \mathbf{L} \boxplus \mathbf{M}}=a^{\prime \mathbf{M}}, a^{\sim \mathbf{L} \boxplus \mathbf{M}}=a^{\sim \mathbf{M}}$. Meets are defined as follows:

$$
a \wedge{ }^{\mathbf{L} \boxplus \mathbf{M}} b=\left\{\begin{array}{l}
a \wedge \wedge^{\mathbf{L}} b \text { if } a, b \in L ; \\
a \wedge \mathbf{M}_{b} \text { if } a, b \in M ; \\
0, \text { otherwise. }
\end{array}\right.
$$

It is not too hard to show that $\mathbf{L} \boxplus \mathbf{M}$ is a $\mathrm{PBZ}^{*}$-lattice, and to acknowledge that our assumption to the effect that at least one of the summands is orthomodular is needed in order to prove that $a \wedge a^{\prime} \leq b \vee b^{\prime}$ for all $a, b \in L \cup M$. The above construction, moreover, can be easily generalised to an arbitrary number of summands $\underset{i \in I}{\bigoplus \mathbf{L}_{i}}$, provided that at most one $\mathbf{L}_{i}$ fails to be orthomodular.

We now proceed with the next 
Definition 4.1. A block of a $P B Z^{*}$-lattice $\mathbf{L}$ is a maximal subalgebra of $\mathbf{L}$ which is either a Boolean algebra or an antiortholattice chain. By $B(\mathbf{L})$ we denote the set of all blocks of L.

We remark that, if $\mathbf{L}=\underset{\mathbf{L}_{i} \in B(\mathbf{L})}{\bigoplus} \mathbf{L}_{i}$, then for every subalgebra $\mathbf{H} \leq \mathbf{L}$ we have that $\mathbf{H}=$ $\bigoplus \mathbf{H}_{i}$. We further denote by $\mathbb{H} \mathbb{P B} \mathbb{Z}^{*}$ the class of subdirectly irreducible PBZ*-lattices $\mathbf{H}_{i} \in B(\mathbf{H})$

which are a horizontal sum of their own blocks, and by $\mathbb{H} \mathbb{P B} \mathbb{Z}_{\text {fin }}^{*}$ the class of finite members of $\mathbb{H} \mathbb{P B} \mathbb{Z}^{*}$. For $a, b \in L$, moreover we let

$$
\gamma(a, b)=(a \vee b) \wedge\left(a \vee b^{\sim}\right) \wedge\left(a^{\sim} \vee b\right) \wedge\left(a^{\sim} \vee b^{\sim}\right)
$$

and we write $a C b$ if $\gamma(a, b)=0$ and $a \bar{C} b$ otherwise.

Lemma 4.1. Let $\mathbf{L}$ be a PBZ*-lattice and let $a \in L$. If $a^{\sim}=0$, then for any $b \in L$, we have that $a C b$.

Proof. In fact, in such a case $\gamma(a, b)=(a \vee b) \wedge\left(a \vee b^{\sim}\right) \wedge b \wedge b^{\sim}=0$.

We now adapt some arguments from [4] to our setting. The next lemma provides necessary and sufficient conditions for a $\mathrm{PBZ}^{*}$-lattice to be representable as horizontal sum of its blocks.

Lemma 4.2. For a $\mathrm{PBZ}^{*}$-lattice $\mathbf{L}$ the following are equivalent:

(1) $\mathbf{L}=\bigoplus_{\mathbf{L}_{i} \in B(\mathbf{L})} \mathbf{L}_{i}$;

(2) L satisfies the conditions:

(a) if $a \bar{C} b$ then $a \vee b=1$;

(b) if $a, b \notin\{0,1\}, a^{\sim}=0$ and $\diamond b=b$, then $a \vee b=1$;

(c) if $a^{\sim}=b^{\sim}=0$, then $a \leq b$ or $b \leq a$;

(d) if $a \notin\{0,1\}$, then $a^{\sim}=0$ or $\diamond a=a$.

Proof. $(1 \rightarrow 2)$

(a) Note that if $a \bar{C} b$, then $a, b$ are sharp elements belonging to different Boolean blocks, and therefore their join is 1 .

(b) If $a, b \notin\{0,1\}, a^{\sim}=0$ and $\diamond b=b$, then $a$ is unsharp and $b$ is sharp, whence they belong to different blocks and thus $a \vee b=1$.

(c) and (d) are obvious. 
$(2 \rightarrow 1)$ If $a \notin S_{K}(L)$, then by (b) $a^{\sim}=0$ and so by Lemma 4.1 we have that, for any $b \in L, a C b$. Therefore, by condition (a) and [4, Lemma 1], the orthomodular subalgebra with universe $S_{K}(L)$ is a horizontal sum of the Boolean blocks of $\mathbf{L}$. Consider the set

$$
U(L)=\{0\} \cup\left\{a \in L: a^{\sim}=0\right\} .
$$

By condition (c), $U(L)$ is linearly ordered and therefore it closed under lattice operations. Now, let $a \in U(L)$ and $0<a<1$, whence $0<a^{\prime}<1$, and suppose further that $a^{\prime \sim} \neq 0$. By condition (d), $\diamond a^{\prime}=a^{\prime}$. Thus $a$ is sharp and so $a^{\prime}=a^{\sim}=0$, a contradiction. Therefore $U(L)$ is closed under ', and then it is the universe of a linearly ordered subalgebra of $\mathbf{L}$ which is an antiortholattice. Finally, the fact that $\mathbf{L}=\bigoplus_{\mathbf{L}_{i} \in B(\mathbf{L})} \mathbf{L}_{i}$ follows from item (b).

If $\mathbf{L}=\bigoplus_{\mathbf{L}_{i} \in B(\mathbf{L})} \mathbf{L}_{i}$ and $\mathbf{L} \notin \mathbb{O M L}$, then for exactly one $i$ we have that $\mathbf{L}_{i}$ is an antiortholattice chain. We now show that $\mathbf{L}$ is s.i. iff such an $\mathbf{L}_{i}=\mathbf{D}_{j}$, for some $3 \leq j \leq 5$.

Lemma 4.3. Let $\mathbf{L}=\underset{\mathbf{L}_{i} \in B(\mathbf{L})}{\bigoplus} \mathbf{L}_{i}$ and $\mathbf{L} \notin \mathbb{O M L}$. Then $\mathbf{L}$ is subdirectly irreducible PBZ*lattice iff its unique non-Boolean summand is either $\mathbf{D}_{3}$ or $\mathbf{D}_{4}$ or $\mathbf{D}_{5}$.

Proof. Suppose first that $\mathbf{L}_{i}$ is not a Boolean algebra and that it is different from $\mathbf{D}_{j}$ for all $3 \leq j \leq 5$, whence $\mathbf{D}_{6} \leq \mathbf{L}_{i}$. Therefore, there is a subchain in $\mathbf{L}_{i}$ having the following form:

$$
0<a<b<b^{\prime}<a^{\prime}<1 \text {. }
$$

It is not hard to check that the equivalences

$$
\begin{aligned}
& \theta_{1}=\left\{(x, y) \in L^{2}: b \leq x, y \leq b^{\prime} \text { or } x=y\right\} \\
& \theta_{2}=\left\{(x, y) \in L^{2}: a \leq x, y \leq b \text { or } b^{\prime} \leq x, y \leq a^{\prime} \text { or } x=y\right\}
\end{aligned}
$$

are nonzero congruences on $\mathbf{L}$, and that $\theta_{1} \cap \theta_{2}=\Delta$. Consequently, $\mathbf{L}$ is not s.i.

Conversely, it suffices to show that $\mathbf{L} \boxplus \mathbf{D}_{j}$ is subdirectly irreducible for all $3 \leq j \leq 5$, and for any orthomodular lattice $\mathbf{L}$. First, we prove that a congruence $\theta<\nabla$ on $\mathbf{L} \boxplus \mathbf{D}_{j}$ cannot identify distinct elements $a, b$ of $L$, for suppose otherwise. Then the congruence class of 0 is nontrivial [3, Lemma 4.2] and there is $0<a<1$ s.t. $a \theta 0$ and $a^{\prime} \theta 1$. Let further $b$ be any element of $D_{j}$ different from 0 and from 1 . We have that

$$
0=a^{\prime} \wedge b \theta 1 \wedge b=b=0 \vee b \theta a \vee b=1,
$$

a contradiction. Next, we prove that $\theta$ cannot identify elements of $L$ with elements of $D_{j}$ either. In fact, if $0<a, b<1, a \in L, b \in D_{j}$ and $a \theta b$, then $0=b^{\sim} \theta a^{\sim}=a^{\prime}$, contradicting what we have previously established. So, $\theta$ can identify at most elements of $D_{j}$. It follows that $\mathbf{L} \boxplus \mathbf{D}_{j}$ is subdirectly irreducible if $\mathbf{D}_{j}$ is such, and this happens for all $3 \leq j \leq 5$.

Let now $H$ be a subclass of $\mathbb{H} \mathbb{P B} \mathbb{Z}_{\text {fin }}^{*}$ that satisfies the following properties:

(a): If $\mathbf{H} \in H$ and $\mathbf{L} \in \mathbb{H} \mathbb{P B} \mathbb{Z}_{\text {fin }}^{*}$ is a subalgebra of $\mathbf{H}$, then $\mathbf{L}$ belongs to $H$; 
(b): If $\mathbf{L} \boxplus \mathbf{D}_{4} \in H$, then $\mathbf{L} \boxplus \mathbf{D}_{3}$ is in $H$.

Let $\mathfrak{L}$ be the complete lattice whose universe is the set of all classes satisfying Conditions (a) and (b) above, lattice-ordered by inclusion.

Theorem 4.1. $\mathfrak{L}$ is isomorphic to the lattice $\mathfrak{H}$ of subvarieties of $V\left(\mathbb{H} \mathbb{P B} \mathbb{Z}^{*}\right)$.

Proof. Let $\phi: \mathfrak{L} \rightarrow \mathfrak{H}$ and $\psi: \mathfrak{H} \rightarrow \mathfrak{L}$ be defined by $\phi(H)=V(H)$ and $\psi(\mathbb{V})=\mathbb{V} \cap \mathbb{H} \mathbb{P} \mathbb{B} \mathbb{Z}_{\text {fin }}^{*}$, respectively. Clearly $\phi, \psi$ are well-defined isotone mappings. We now show that $\psi \circ \phi=\mathrm{id}_{\mathfrak{L}}$. It can be readily seen that $H \subseteq \psi \circ \phi(H)=V(H) \cap \mathbb{H} \mathbb{P} \mathbb{Z}_{\text {fin }}^{*}$. Let $\mathbf{L} \in V(H) \cap \mathbb{H} \mathbb{P B} \mathbb{Z}_{\text {fin }}^{*}$, and suppose that $\mathbf{L} \notin H$. Since $\mathbf{L}$ is in $\mathbb{H} \mathbb{P B} \mathbb{Z}_{f i n}^{*}, \mathbf{L}$ is subdirectly irreducible, and because it is also in $V(H)$, by Jónsson's Lemma $\mathbf{L}$ is in $H S P_{u}(H)$. Let us note that not being a subalgebra of any $\mathbf{H}$ in $H$ is a first order property. Furthermore, being a horizontal sum of one's blocks is again a first order property, by Lemma 4.2. Then, every algebra in $S P_{u}(H)$ is a horizontal sum of this sort. If $\mathbf{L}$ has one block, then $\mathbf{L}$ is either the two-element Boolean algebra, or by Lemma 4.3 is either $\mathbf{D}_{3}, \mathbf{D}_{4}$ or $\mathbf{D}_{5}$, and in both cases it belongs to $H$. Otherwise, by Lemma 4.3 again, $\mathbf{L}$ is the horizontal sum of its Boolean blocks and $\mathbf{D}_{j}$, for some $3 \leq j \leq 5$. Again, $\mathbf{L}$ will be in $H$. Therefore, $\psi \circ \phi(H)=H$.

As regards the converse, it is evident that if $\mathbb{V} \in \mathfrak{H}$, then $\mathbb{V} \supseteq \phi \circ \psi(\mathbb{V})=H S P(\mathbb{V} \cap$ $\mathbb{H} \mathbb{P B} \mathbb{Z}_{\text {fin }}^{*}$ ). For the converse inclusion, let $\mathbb{V}$ be an element of $\mathfrak{H}$, and $\mathbf{L}$ be subdirectly irreducible in $\mathbb{V}$. Then, $\mathbf{L}$ is the horizontal sum of its Boolean blocks and an antiortholattice chain. If $\mathbf{H}$ is a finitely generated subalgebra of $\mathbf{L}$, then $\mathbf{H}$ is finite because it is the horizontal sum of its Boolean blocks and an antiortholattice chain, which are both locally finite. Then, either $\mathbf{H}$ is Boolean, or $\mathbf{H}$ is in $\mathbb{V} \cap \mathbb{H} \mathbb{P B} \mathbb{Z}_{\text {fin }}^{*}$. In both cases, $\mathbf{H}$ is contained in $H S P\left(\mathbb{V} \cap \mathbb{H} \mathbb{P B} \mathbb{Z}_{\text {fin }}^{*}\right)$. This is the case for any finitely generated subalgebra of $\mathbf{L}$. Therefore, L itself belongs to $H S P\left(\mathbb{V} \cap \mathbb{H} \mathbb{P B} \mathbb{Z}_{\text {fin }}^{*}\right)$.

A direct consequence of Theorem 4.1 is the following:

Corollary 4.1. Every subvariety of $V\left(\mathbb{H} \mathbb{P B} \mathbb{Z}^{*}\right)$ is generated by its finite members.

Acknowledgement. R. Giuntini gratefully acknowledges the support of the Regione Autonoma Sardegna within the project "Modeling the uncertainty: quantum theory and imaging processing", LR 7/8/2007, RAS CRP-59872. All authors gratefully acknowledge the support of the Horizon 2020 program of the European Commission: SYSMICS project, Proposal Number: 689176, MSCA-RISE-2015, and the suggestions of two referees.

\section{REFERENCES}

[1] Bergmann M., An Introduction to Many-Valued and Fuzzy Logic, Cambridge University Press, Cambridge, 2008.

[2] Birkhoff G., Lattice Theory, AMS Colloquium Publications, New York, 1948.

[3] Bruns G., Harding J., "Algebraic aspects of orthomodular lattices", in B. Coecke et al. (Eds.), Current Research in Operational Quantum Logic, Springer, Berlin, 2000, pp 37-65. 
[4] Bruns G., Kalmbach G., "Varieties of orthomodular lattices", Canadian Journal of Mathematics, 5, 1971 , pp. $802-810$.

[5] Cattaneo G., Nisticò G., "Brouwer-Zadeh posets and three-valued Łukasiewicz posets", Fuzzy Sets and Systems, 33, 2, 1989, pp. 165-190.

[6] Chajda I., "A note on pseudo-Kleene algebras", Acta Univ. Palacky Olomouc, 55, 1, 2016, pp. 39-45.

[7] Chajda I., Länger H., "Horizontal sums of bounded lattices", Mathematica Pannonica, 20, 1, 2009, pp. $1-5$.

[8] Cignoli R., "The class of Kleene algebras satisfying an interpolation property and Nelson algebras", Algebra Universalis, 23, 1986, pp. 262-292.

[9] Dalla Chiara M.L., Giuntini R., Greechie R.J., Reasoning in Quantum Theory, Kluwer, Dordrecht, 2004.

[10] Dvurečenskij A., Olson order of quantum observables, International Journal of Theoretical Physics, 55, 2016, pp. 4896-4912.

[11] Giuntini R., "Three-valued Brouwer-Zadeh logic", International Journal of Theoretical Physics, 32, 1993, pp. 1875-1887.

[12] Giuntini R., Ledda A., Paoli F., "A new view of effects in a Hilbert space", Studia Logica, 104, 2016, pp. 1145-1177.

[13] Greechie R.J., "On the structure of orthomodular lattices satisfying the chain condition", Journal of Combinatorial Theory, 4, 3, 1968, pp. 210-218.

[14] de Groote H.F., "On a canonical lattice structure on the effect algebra of a von Neumann algebra", arXiv:math-ph/0410018v2, 2005.

[15] Harzheim E., Ordered Sets, Springer, Berlin, 2005.

[16] Kalman J.A., "Lattices with involution", Transactions of the American Mathematical Society, 87, 1958, pp. 485-491.

[17] Kreyszig E., Introductory Functional Analysis with Applications, Wiley, New York, 1978.

[18] Olson M.P., "The self-adjoint operators of a von Neumann algebra form a conditionally complete lattice", Proceedings of the American Mathematical Society, 28, 1971, pp. 537-544.

[19] Stroock D.W., A Concise Introduction to the Theory of Integration (3rd ed.), Birkhäuser, Basel, 1998.

University of CAGLiARI 